OPEN ACCESS

Edited by:

Mario Luciano,

University of Campania Luigi

Vanvitelli, Italy

Reviewed by:

Gaia Sampogna,

University of Campania "L

Vanvitelli", Italy

Luca Steardo,

University Magna Graecia of

Catanzaro, Italy

*Correspondence:

Antonio Ventriglio

a.ventriglio@libero.it

Specialty section

This article was submitted to

Psychosomatic Medicine,

a section of the journal

Frontiers in Psychiatry

Received: 29 October 2021 Accepted: 23 November 2021 Published: 15 December 2021

Citation:

Ventriglio A, Petito A

Castaldelli-Maia JM, Torales J, Sannicandro V, Milano E, luso $S$ and

Bellomo A (2021) Use of Psychoeducation for Psychotic Disorder Patients Treated With Modern, Long-Acting, Injected Antipsychotics.

Front. Psychiatry 12:804612. doi: 10.3389/fpsyt.2021.804612

\section{Use of Psychoeducation for Psychotic Disorder Patients Treated With Modern, Long-Acting, Injected Antipsychotics}

\author{
Antonio Ventriglio ${ }^{1 *}$, Annamaria Petito ${ }^{1}$, João Maurício Castaldelli-Maia ${ }^{2}$, Julio Torales ${ }^{3}$, \\ Valeria Sannicandro ${ }^{1}$, Eleonora Milano ${ }^{1}$, Salvatore Iuso $^{1}$ and Antonello Bellomo ${ }^{1}$ \\ ${ }^{1}$ Department of Clinical and Experimental Medicine, University of Foggia, Foggia, Italy, ${ }^{2}$ Department of Neuroscience, \\ Medical School, Fundação do ABC, Santo André, Brazil, ${ }^{3}$ Department of Psychiatry, School of Medical Sciences, National \\ University of Asunción, Asunción, Paraguay
}

Introduction: There is an increased risk of adverse metabolic effects of some modern antipsychotic drugs, and concern that long-acting, injected preparations of them may increase such risk. We now report on clinical and metabolic outcomes in patient-subjects diagnosed with affective and non-affective psychotic disorders following exposure to psychoeducation on metabolic risks of modern antipsychotics followed by treatment with long-acting atypical injected antipsychotics over 6-months.

Materials and Methods: 85 psychotic disorder outpatients (42 affective [AP]; 43 non-affective [NAP]) at the University of Foggia were treated with long-acting, injected, second-generation antipsychotics in association with a set of psychoeducational sessions concerning general health and potential effects of antipsychotic drug treatments. They were evaluated at baseline and six months.

Results: Initially, NAP subjects reported higher ratings of positive and negative symptoms than AP subjects, were more likely to receive risperidone or paliperidone, with higher CPZ-eq doses of antipsychotics (294.0 \pm 77.8 vs. $229.3 \pm 95.8$ $\mathrm{mg} /$ day), and shorter QTc electrocardiographic recovery intervals. During the 6-month follow-up, ratings of treatment-adherence improved through overall $(+8.75 \%)$, and symptom-ratings decreased (-7.57\%) as did Body-Mass Index (-2.40\%; all $p \leq 0.001)$. Moreover, serum levels of fasting glucose, hemoglobin glycosylation, cholesterol and prolactin concentrations all decreased, with little difference between subjects with AP vs. NAP.

Discussion and Conclusions: A psychoeducational program was associated with consistent improvement in psychotic symptoms and several metabolic and physiological measures, as well as with treatment-adherence during six months of treatment with long-acting, injected, second-generation antipsychotics, in association with both affective and non-affective psychotic disorders.

Keywords: long-acting antipsychotics, psychotic disorders, metabolic changes, psychoeducation, treatmentadherence 


\section{INTRODUCTION}

Major mental disorders are associated with increased risk of morbidity and mortality due to the illnesses themselves and their treatments (1). The prevalence of metabolic syndrome (MS) among schizophrenia patients may range 37-63\% with a relative risk (RR) of $2-3$ in patients compared to general population, as well as MS in bipolar disorder patients ranges $30-49 \%$ with a RR of $1.5-2$ (2). Consequently, it has been largely described an increased risk of death from medical causes in schizophrenia and 20\% (10-15 years) shorter lifespan $(2,3)$; similarly, affective psychoses are associated with higher standardized mortality ratios from medical causes than general population ranging 1.9-2.1 (2, 3). Many factors including poor life-style and food intake, poor attention to health needs and side effects of psychotropic drugs may impact on the metabolic outcome of patients affected by psychoses (3). Currently, the employment of second- and third- generation antipsychotics, such as serotonin- dopamine antagonists or dopamine partial agonists, are clinically preferred since are more effective on negative, affective and cognitive symptoms of psychosis (4). Nonetheless, newer antipsychotics may induce increases in bodyweight, insulin- resistance with long- term adverse physiological effects including type-2- diabetes mellitus, hypertension and dyslipidemia, in addition to obesity (4-6). Also, it has been largely described that the prevalence of metabolic syndrome in patients treated with antipsychotics is approximately $40 \%$ (33.8-42.1\%) in contrast to rates of $10.4-12.2 \%$ among psychosis un-medicated patients: some atypical antipsychotics such as olanzapine and risperidone were associated to higher weight gain among non-clozapine second generation antipsychotics, as well as risperidone and amisulpride were responsible for increasing serum prolactin (7).

These metabolic issues are potentially life-threatening effects and need to be carefully assessed and treated by clinicians $(8,9)$. Also, strategies of prevention need to be employed and awareness regarding their own general health needs to be addressed among these patients (10). International organizations have proposed guidelines for screening and preventing metabolic issues in patients treated with antipsychotics suggesting physical monitoring and psycho-education in the long-term treatment $(11,12)$.

Physical activity may be a relevant therapeutic intervention for people with severe mental disorders and life style-related medical issues: this has been confirmed among schizophreniaspectrum disorders patients as well as those affected by major depressive disorder and bipolar disorder (2). In addition, lifestyle factors such as tobacco smoking, unhealthy dietary patterns, poor sleep, together with poor physical activity have been found to be associated to higher risk of mental illness and poorer outcome of illness: these data suggest that life-style factors need to be addressed within mental health care (12).

We describe the impact of a repeated, systematic, prospective psycho- educational intervention on clinical and metabolic outcomes of patients affected by stable affective (APs) and nonaffective psychoses (NAPs) treated with long-acting atypical antipsychotics over 6-months. We compared psychopathology and medical parameters of interest between the diagnostic groups at baseline and at the end of the program aimed at increasing awareness of the general health, improving diet and exercise, and limiting obesity and other side effects.

\section{MATERIALS AND METHODS}

\section{Subjects}

85 outpatients affected by Affective Psychoses ( $n=42$; APs: Schizoaffective Disorders, Not Otherwise Specified Psychoses) and Non-Affective Psychoses ( $n=43$; NAPs: Schizophrenia, Delusional Disorders) attending the Unit of Psychiatric at University of Foggia and treated with long-acting atypical antipsychotics, at stable doses, have been recruited and assessed at intake (T1) and 6 months (T2) for psychopathology, treatments, adherence, and monitored for anthropometric and electrocardiographic measures. Also, they received a systematized, repeated psycho-education about physical health. Diagnoses met DSM-5 (The Diagnostic and Statistical Manual of Mental Disorders, Fifth Edition), confirmed with the MiniInternational Neuropsychiatric Interview (MINI) performed by consensus of experienced clinicians (AV, AP, and SI) $(13,14)$. This is a real-world study based on a straightforward clinical assessment with easy and reliable tools.

Patients were voluntarily recruited, clinically treated and followed at the Psychiatric Outpatient Services of University of Foggia Medical Center in 2014-2018, as part of an approved PhD Program in Clinical and Experimental Medicine at University of Foggia. All participants provided written, informed consent and the intervention was approved by the Medical Center Ethical Review Committee. Acutely psychiatrically or medically ill patients were excluded and we recruited those reporting a stable phase of illness in the previous three months and treated with a stable long-acting antipsychotic treatment clinically determined and followed for six months.

\section{Assessment and Monitoring Procedures}

Patients were assessed and monitored at intake (T1) and 6 months (T2) of follow-up, employing standardized methods. Clinical assessment, treatment selection and physical monitoring have been run by psychiatrists ( $\mathrm{AV}, \mathrm{AB}, \mathrm{VS}, \mathrm{EM})$, whereas psychoeducation and rating scales have been delivered by two expert psychologists (AP, SI). Required information was gathered at intake and repeatedly during the follow-up. Electrocardiograms (with the record of QTc interval) were performed at intake and final visit as well as anthropometrics (height, weight, waist circumference, body mass index [BMI]), vital signs (blood pressure and pulse rate) and serum assays (serum lipids, carbohydrates, prolactin) which were carried out consistently by the University Medical Center clinical laboratory.

Antipsychotic drug- doses were converted to $\mathrm{mg} /$ day chlorpromazine-equivalents according to suggested conversion formulas $(15,16)$. Adherence to treatment was rated with the 30-item Drug Attitude Inventory (DAI-30) at intake and six months (17). Psychopathology including positive, negative and general symptoms were rated with the Positive and Negative Syndrome Scale (PANSS) (18) and the Brief Psychiatric 
Rating Scale (BPRS) (19) at intake and six months; each investigator has been trained in order to employ the tools correctly and levels of inter-rater agreement were calculated $(\kappa$-statistic $\geq 0.94)$.

\section{Psychoeducational Program}

The repeated, systematic, prospective psycho- educational intervention was provided to all subjects once/month in conjunction with the administration of monthly long-acting injectable treatment. It included six sessions concerning psychiatric and general health, diet, exercise, weight-control, current treatment, following methods recommended by Littrell and colleagues (20). Patients were also trained to regularly measure vital signs (blood pressure, pulse rate) anthropometric parameters (weight, body-mass index [BMI], waist circumference) and advised about food-selection, healthy diets, daily physical exercise. Motivational modules regarding daily activity were performed.

In this trial no control condition was included.

\section{Data Analysis}

Data analyses were performed with standard, commercial, statistical software (Statview ${ }^{\circledR}$; SAS Institute, Cary, NC; Stata ${ }^{\circledR}$, StataCorp, College Station, TX). Data were presented as means or percentages (including \%-changes) with 95\%- Confidence Intervals- or standard deviations (as \%-changes), compared between diagnosis by $\boldsymbol{t}$-test or $\chi^{2}$. We also carried out repeated-measures ANOVA to evaluate changes in parameters of interest over time. Findings are considered statistically significant with two-tailed $p \leq 0.05$.

TABLE 1 | Subject characteristics at intake.

\begin{tabular}{|c|c|c|c|c|}
\hline \multirow[t]{2}{*}{ Measures } & \multicolumn{3}{|c|}{ Means $[95 \% \mathrm{Cl}]$} & \multirow[t]{2}{*}{$p$-value } \\
\hline & Non-affective psychoses & Affective psychoses & All pychoses & \\
\hline Number (n) & 43 & 42 & 85 & - \\
\hline Female (\%) & $30.2[17.2-46.1]$ & $52.4[36.4-68.0]$ & $41.2[30.6-52.4]$ & 0.048 \\
\hline Age (years) & $43.8[39.6-48.0]$ & $40.3[36.7-43.9]$ & $42.0[39.3-41.7]$ & 0.212 \\
\hline Married (\%) & 9.30 [2.59-22.1] & 21.4 [10.3-36.8] & $15.3[8.40-24.7]$ & 0.142 \\
\hline Employed (\%) & $6.98[1.46-19.9]$ & $19.1[8.60-34.1]$ & $12.9[6.64-22.0]$ & 0.117 \\
\hline \multicolumn{5}{|l|}{ Initial morbidity ratings } \\
\hline PANSS (total) & $63.2[53.9-72.5]$ & $50.4[44.7-56.1]$ & $56.9[51.4-62.4]$ & 0.022 \\
\hline BPRS (total) & $42.3[37.9-46.7]$ & $36.9[33.1-40.7]$ & 39.6 36.7-42.5[] & 0.068 \\
\hline \multicolumn{5}{|l|}{ Antipsychotic use (\%) } \\
\hline Risperidone-LAl & $27.9[15.3-43.7]$ & $9.52[2.66-22.6]$ & $18.8[11.2-28.8]$ & 0.030 \\
\hline Paliperidone-LAI & $72.1[56.3-84.7]$ & $42.9[27.7-59.0]$ & $57.6[46.4-68.3]$ & $<0.001$ \\
\hline Olanzapine-LAl & $0.00[0.00-0.00]$ & $16.7[6.97-31.4]$ & $8.24[3.38-16.2]$ & 0.005 \\
\hline Aripiprazole-LAl & $0.00[0.00-0.00$ & $31.0[17.6-31.4]$ & $15.3[8.40-24.7]$ & $<0.001$ \\
\hline Mean dose (CPZ-eq mg/day) & 294 [270-318] & 229 [199-259] & 262 [242-282] & 0.001 \\
\hline DAl-30 score & $10.9[10.2-11.6]$ & $11.1[10.4-11.8]$ & $11.0[10.5-11.5]$ & 0.765 \\
\hline Body-mass index (BMl, kg/m²) & $28.4[26.7-30.1]$ & $28.1[26.0-30.2]$ & $28.2[26.9-29.5]$ & 0.792 \\
\hline Waist circumference (cm) & 103 [98.3-108] & $101[95.7-106]$ & $102[98.5-105]$ & 0.668 \\
\hline \multicolumn{5}{|l|}{ Blood pressure \& pulse } \\
\hline Systolic (mm Hg) & 119 [116-122] & 122 [118-126] & $118[116-120]$ & 0.262 \\
\hline Diastolic (mm Hg) & $76.8[74.2-79.4]$ & 75.3 [72.4-78.2] & 76.3 [74.4-78.2] & 0.191 \\
\hline Pulse rate (per min) & 85.7 [82.6-88.8] & $83.4[79.6-85.6]$ & $83.4[81.5-85.3]$ & 0.399 \\
\hline QTc interval (msec) & 407 [398-416] & 420 [413-427] & $413[407-419]$ & 0.020 \\
\hline \multicolumn{5}{|l|}{ Carbohydrates (serum) } \\
\hline Fasting blood glucose & 94.7 [90.1-99.3] & 94.3 [89.4-99.2] & $94.5[91.2-97.8]$ & 0.897 \\
\hline Hemoglobin glycosylation (\%) & $6.28[6.02-6.55]$ & $6.15[5.96-6.34]$ & $6.09[6.03-6.15]$ & 0.442 \\
\hline \multicolumn{5}{|l|}{ Serum lipids (mg/dL) } \\
\hline Triglycerides & $143[124-162]$ & 150 [120-180] & $146[129-163]$ & 0.710 \\
\hline Total cholesterol & 188 [178-198] & 196 [184-208] & 192 [184-200] & 0.335 \\
\hline Low-density cholesterol (LDL) & 125 [117-133] & $123[110-136]$ & $124[117-131]$ & 0.859 \\
\hline High-density cholesterol (HDL) & 45.9 [42.5-49.3] & $49.8[45.7-54.3]$ & $47.8[45.1-50.5]$ & 0.150 \\
\hline Serum prolactin ( $\mu \mathrm{g} / \mathrm{L})$ & $45.1[34.6-55.6]$ & 40.4 [27.9-52.9] & $42.8[34.8-50.8]$ & 0.563 \\
\hline
\end{tabular}

Boldface: Factors significantly different at intake by diagnostic type, by t-test or $\chi^{2}(p<0.05)$.

PANSS, Positive and Negative Syndrome Scale; BPRS, Brief Psychiatric Rating Scale; CPZ-eq, chlorpromazine-equivalent mg/day; DAl-30, 30-item Drug Attitude Inventory. 


\section{RESULTS}

\section{Sample Characteristics at Intake}

85 adult outpatients affected by Affective Psychoses $(n=$ 42; APs: Schizoaffective Disorders, Not Otherwise Specified Psychoses $)$ and Non-Affective Psychoses $(n=43$; NAPs: Schizophrenia, Delusional Disorders) were recruited in the 20142018 period, treated and followed for six months. Those with NAPs or APs included 30.2 and $52.3 \%$ women respectively $(p=0.048)$, with overall age-at-intake of $43.8 \pm 12.2$ vs. $40.3 \pm 11.6$ years. Demographic characteristics were similar through the diagnostic subgroups (Table 1). Psychopathological symptoms rated with PANSS and BPRS scales were slightly higher among NAPs vs. APs patients $(63.2 \pm 30.3$ vs. $50.4 \pm$ 18.2 [PANSS; $p=0.022$ ] and $42.3 \pm 14.4$ vs. $36.9 \pm 12.3$ [BPRS; $p=0.068]$ ).

Long-acting treatments, clinically selected, were exclusively based on SDA agents (injectable- risperidone [27.9\%] and - paliperidone [72.0\%]) for NAP patients and also included olanzapine and aripiprazole for AP patients ranking: paliperidone $(42.8 \%)>$ aripiprazole $(30.9 \%)>$ olanzapine $(16.6 \%)>$ risperidone $(9.52 \%) \quad(p=0.000)$. Average daily doses of antipsychotic (all converted to chlorpromazineequivalent milligrams for comparison) were much higher among NAP vs. AP patients $(294.0 \pm 77.8$ vs. $229.3 \pm 95.8$; $p=0.001)$.

Baseline anthropometrics, serum parameters and vital signs were somewhat similar between diagnostic groups and recorded QTc electrocardiographic interval was slightly higher among AP vs. NAP patients $(419.9 \pm 22.3$ vs. $406.6 \pm 29.2 ; p=0.020)$ (Table 1).

\section{Follow-Up Assessment}

Measures changes from baseline (T1) were recorded after 6 months (T2) of follow-up and psycho- educational program. Changes were computed as means \pm standard deviations (not shown) but also presented in a more informative manner as \%changes with standard deviations: [(parameter at T1-parameter at T2)/ parameter at $T 1]^{*} 100$. Findings for all cases $(N=85)$ at 6-months showed a significant improvement of adherencerate (DAI-30; $+8.75 \%)$, reduction of psychopathological ratings $(-7.57 \%$ at PANSS and $-6.45 \%$ at BPRS), decrease of BMI $(-2.40 \%)$ as well as QTc Interval $(-0.20 \%)$, fasting glucose $(-2.54 \%)$, hemoglobin glycosylation $(-3.47 \%)$, total cholesterol with an increase of high density lipoproteins $(-3.98$ and +34.8 $\%$ respectively), reduction in serum prolactin $(-4.81 \%)(0.000 \leq p$ $\leq 0.002$ ). Measures changes have shown little differences among diagnoses. PANSS score decreased highly among NAP vs. AP patients ( -9.54 vs. $-3.76 \%)$ as well as hemoglobin glycosylation ( -3.84 vs. $-2.76 \%)$, QTc interval $(-0.209$ vs. $-0.203 \%)$ and serum prolactin $(-4.97$ vs. $-4.49 \%)(0.006 \leq p \leq 0.035)$ (Table 2). Finally, we computed changes in measures among the different long-acting treatments over time: all measures did not differ among the treatments $(0.068 \leq p \leq 0.981$; not shown).

\section{DISCUSSION AND CONCLUSION}

This study aimed to test the impact of a psychoeducational program on general health among 85 patient-subjects diagnosed with clinically stable affective and non-affective psychoses treated with long-acting, injected, second-generation drugs,

TABLE 2 | Changes of measures over six months of long-acting antipsychotic treatment and psychoeducation.

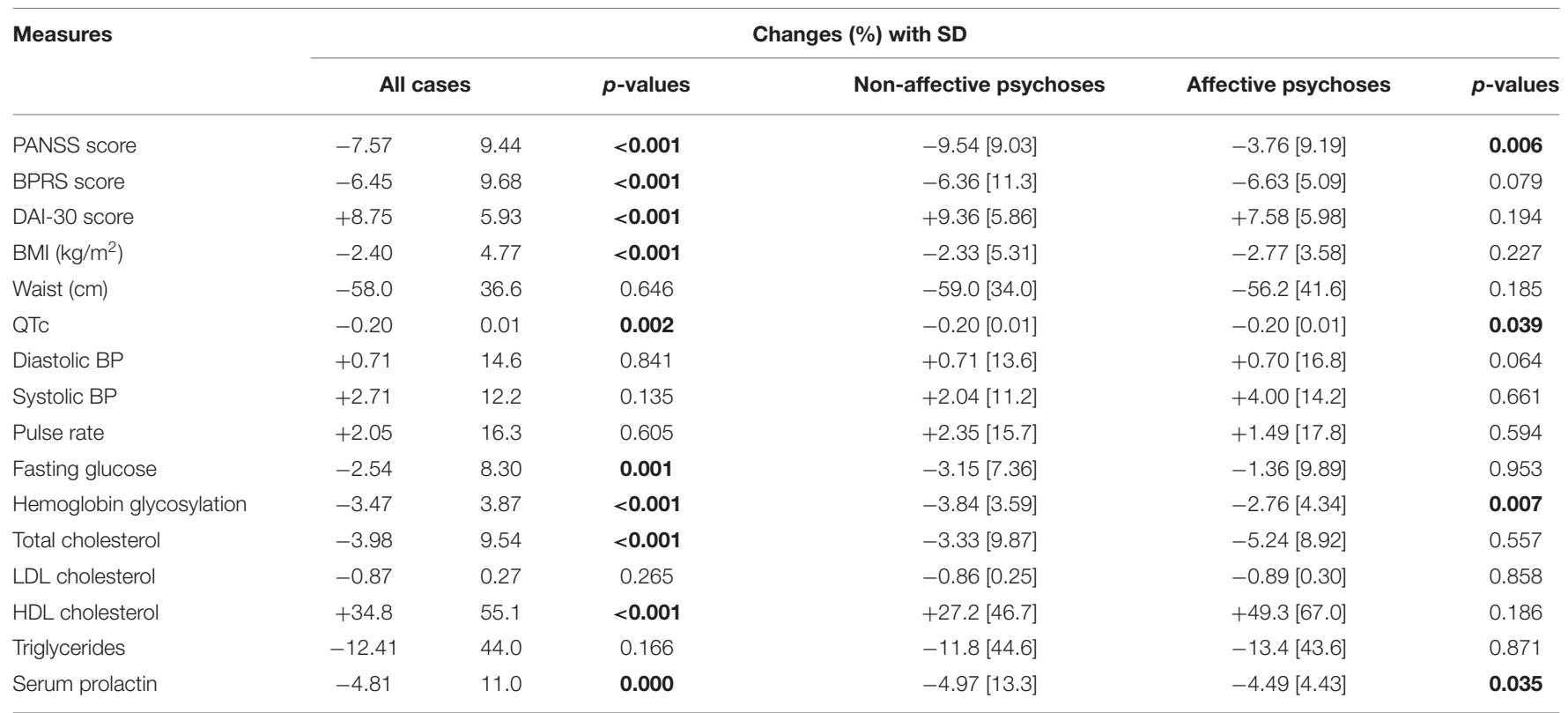

The 85 APs and NAPs patient-subjects were assessed clinically at baseline and 6 months, changes in measures were tested by repeated measures ANOVA.

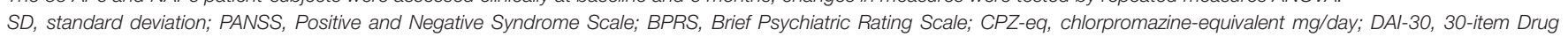
Attitude Inventory; BMI, Body-Mass Index; HDL, High-Density Lipids (cholesterol). LDL, Low-Density Lipids.

Boldface: Factors significantly different. 
as determined by treating physicians. Changes in clinical, anthropometric and physiological measures over six months of treatment exposure were measured.

Clinicians preferred risperidone and paliperidone antipsychotics for the long-term treatment of patient-subjects diagnosed with schizophrenia or other non-affective psychoses, possibly due to their relatively high affinity at dopamine $\mathrm{D}_{2}$-receptors and their reported benefits on both positive and negative psychotic symptoms (21). Olanzapine and aripiprazole were preferred with affective psychoses since they may have mood-stabilizing effects (22). Subjects with non-affective psychoses were also given relatively high CPZ-eq doses of antipsychotics. Among few initial differences between subjects with affective and non-affective disorders, the QTc electrocardiographic interval was slightly higher with affective disorders (Table 1). It is of interest that patients recruited in the study were not affected by serious cardiological conditions and a small number of subjects were taking anti-hypertensive drugs (27.2\%; not described for heterogeneity of data). Variations of QTc intervals among patients may also reflect an additional individual variability (23).

By six-months of treatment that involved close clinical followup and continued psychoeducational intervention was associated with several moderate, but favorable changes (Table 2). These include improved adherence ratings, even above those expected with injected, long-acting drugs: this reflects the evidence that joining a psycho-educational program may increase the personal awareness about illness and improve patients' attitudes regarding its treatment (24). Also, improvements in psychoticsymptoms ratings (PANSS and BPRS) may reflect benefits of the medication provided and perhaps added benefits associated with the psychoeducational intervention aimed to increase patients' insight (25). Improvements in BMI, carbohydrates, hemoglobin glycosylation, serum lipids and prolactin concentration may in part reflect changes in life-style, diet and physical exercise, all as encouraged by the psychoeducation intervention (26). In addition, participants' attitudes toward medications and their adherence to scheduled injections improved as reflected in ratings with the DAI- 30 .

\section{Limitations}

Cause-and-effect relationships involved in the observed changes are not clear without a control condition lacking

\section{REFERENCES}

1. Hert DEM, Correll CU, Bobes J, Cetkovich-Bakmas M, Cohen D, Asai I, et al. Physical illness in patients with severe mental disorders. I. Prevalence, impact of medications and disparities in health care. World Psychiatry. (2011) 10:52-77. 10.1002/j.2051-5545.2011.tb00014.x

2. Stubbs B, Vancampfort D, Hallgren M, Firth J, Veronese N, Solmi $\mathrm{M}$, et al. EPA guidance on physical activity as a treatment for severe mental illness: a meta-review of the evidence and Position Statement from the European Psychiatric Association (EPA), supported by the International Organization of Physical Therapists in Mental Health (IOPTMH). Eur Psychiatry. (2018) 54:124-44. doi: 10.1016/j.eurpsy.2018. 07.004 psychoeducation. In fact, the comparison with a controlgroup of patients treated with long-acting medications with no psychoeducation (as well as patients treated with oral antipsychotics and psychoeducation) would add more evidence on the cause-and-effect relationships between their clinical outcomes and psychoeducational intervention. Also, it is notable that differences in baseline measures and their changes with treatment related to the type of psychotic illness were somewhat negligible (Tables 1, 2). This study is limited by the relatively small number of subjects, only 6 months of treatment and follow-up, the lack of a comparison condition without a psychoeducational component, and lack of blinding to diagnosis and treatment. Nonetheless, similar improvements in clinical and metabolic status of severe mentally ill patients given the same psycho-educational program have been confirmed in our previous study conducted in 2014 (3).

In conclusion, the findings suggest favorable changes in clinical and metabolic status among severely ill psychotic outpatients treated with modern, long-acting, injected antipsychotic drugs combined with close clinical follow-up and ongoing psychoeducation. We suggest that such interventions may contribute to improving clinical and medical outcomes in psychotic disorders and limit mortality-risk, and conclude that they require further, controlled testing.

\section{DATA AVAILABILITY STATEMENT}

The raw data supporting the conclusions of this article will be made available by the authors, without undue reservation.

\section{ETHICS STATEMENT}

This study involving human participants has been reviewed and approved by University of Foggia. The patients/participants provided their written informed consent to participate in this study.

\section{AUTHOR CONTRIBUTIONS}

$\mathrm{AV}$ and $\mathrm{AB}$ : each authors has contributed to the development of the report. AV, AP, VS, EM, and SI: reviewing clinical and pharmacy records. AV, JC-M, and JT: analyzing data. All authors contributed to the article and approved the submitted version. 
6. Bartoli F, Crocamo C, Caslini M, Clerici M, Carrà G. Schizoaffective disorder and metabolic syndrome: A meta-analytic comparison with schizophrenia and other non-affective psychoses. J Psychiatr Res. (2015) 66-67:12734. doi: 10.1016/j.jpsychires.2015.04.028

7. Kishimoto T, Hagi K, Nitta M, Kane JM, Correll CU. Long-term effectiveness of oral second-generation antipsychotics in patients with schizophrenia and related disorders: a systematic review and meta-analysis of direct head-to-head comparisons. World Psychiatry. (2019) 18:20824. doi: 10.1002/wps.20632

8. Ventriglio A, Gentile A, Stella E, Bellomo A. Metabolic issues in patients affected by schizophrenia: clinical characteristics and medical management. Front Neurosci. (2015) 9:297. doi: 10.3389/fnins.2015. 00297

9. Ventriglio A, Baldessarini RJ, Vitrani G, Bonfitto I, Cecere AC, Rinaldi A, et al. Metabolic syndrome in psychotic disorder patients treated with oral and long-acting injected antipsychotics. Front Psychiatry. (2019) 9:744. doi: 10.3389/fpsyt.2018.00744

10. Kelly JR, Gounden P, McLoughlin A, Legris Z, O'Carroll T, McCafferty $\mathrm{R}$, et al. Minding metabolism: targeted interventions to improve cardiometabolic monitoring across early and chronic psychosis. Ir J Med Sci. (2021) 8:1-10. doi: 10.1007/s11845-021-02576-5

11. De Hert M, Dekker JM, Wood D, Kahl KG, Möller HJ. Cardiovascular disease and diabetes in people with severe mental illness position statement from the European Psychiatric Association (EPA) with the European Association for the Study of Diabetes (EASD) and the European Society of Cardiology (ESC). Eur Psychiatry. (2009) 24:412-24. doi: 10.1016/S1888-9891(09) 70714-1

12. Firth J, Solmi M, Wootton RE, Vancampfort D, Schuch FB, Hoare E, et al. A meta-review of "lifestyle psychiatry": the role of exercise, smoking, diet and sleep in the prevention and treatment of mental disorders. World Psychiatry. (2020) 19:360-3809. doi: 10.1002/wps.20773

13. American Psychiatric Association (APA). Diagnostic and Statistical Manual of Mental Disorders - Fifth edition (DSM-5). Washington, DC: Am Psychiat Pub. (2013).

14. Sheehan DV, Lecrubier Y, Sheehan KH, Amorim P, Janavs J, Weiller E, et al. Mini-International Neuropsychiatric Interview (MINI): development and validation of a structured diagnostic psychiatric interview for DSM-IV and ICD-10. J Clin Psychiatry. (1998) 59:22-33.

15. Baldessarini RJ, Tarazi FI. Drugs and the treatment of psychiatric disorders: antipsychotic and antimanic agents. In: Hardman JG, Limbird LE, Gilman AG, editors Goodman and Gilman's the pharmacological basis of therapeutics 11th ed. New York: McGraw-Hill. (2005).

16. Gardner DM, Murphy AL, O’Donnell H, Centorrino F, Baldessarini RJ. Equivalent doses of antipsychotic agents: findings from an international Delphi survey. Am J Psychiatry. (2010) 167:686-93. doi: 10.1176/appi.ajp.2009.09060802

17. Hogan TP, Awad AG, Eastwood R. A self-report scale predictive of drug compliance in schizophrenics: reliability and discriminative validity. Psychol Med. (1983) 13:177-83. doi: 10.1017/s0033291700050182

18. Kay SR, Fiszbein A, Opler LA. The positive and negative syndrome scale (PANSS) for schizophrenia. Schizophrenia Bull. (1987) 13:26176. doi: 10.1093/schbul/13.2.261
19. Dingemans PM, Linszen DH, Lenior ME, Smeets RM. Component structure of the expanded Brief Psychiatric Rating Scale (BPRS- E). Psychopharmacol. (1995) 122:263-7. doi: 10.1007/BF02246547

20. Littrell KH, Hilligoss NM, Kirshner CD, Petty RG, Johnson CG. The effects of an educational intervention on antipsychotic-induced weight gain. J Nursing Scholarsh. (2003) 35:237-41. doi: 10.1111/j.1547-5069.2003.00237.x

21. Lupták M, Michaličková D, Fišar Z, Kitzlerová E, Hroudová J. Novel approaches in schizophrenia-from risk factors and hypotheses to novel drug targets. World J Psychiatry. (2021) 11:277-96. doi: 10.5498/wjp.v11.i7.277

22. McGirr A, Vöhringer PA, Nassir Ghaemi S, Lam RW, Yatham LN. Safety and efficacy of adjunctive second-generation antidepressant therapy with a mood stabiliser or an atypical antipsychotic in acute bipolar depression: randomised systematic review and meta-analysis of a placebo-controlled trials. Focus (Am Psychiatr Publ). (2021) 19:129-37. doi: 10.1176/appi.focus.19102

23. Mangona E, Sandonato E, Brothers TN, Pawasauskas J. Drug-induced QTc prolongation: what we know and where we are going. Curr Drug Saf. (2021). doi: 10.2174/1574886316666210922153059

24. Sampogna G, Luciano M, Del Vecchio V, Giallonardo V, Palummo C, Andriola I, et al. Stili di vita nei pazienti con disturbi mentali gravi: quali opportunità per il trattamento? [Lifestyle in patients with severe mental disorders: a new target for psychosocial interventions?]. Riv Psichiatr. (2021) 56:26171. doi: $10.1708 / 3681.36674$

25. Bighelli I, Rodolico A, García-Mieres H, Pitschel-Walz G, Hansen WP, Schneider-Thoma J, et al. Psychosocial and psychological interventions for relapse prevention in schizophrenia: a systematic review and network meta-analysis. Lancet Psychiatry. (2021) S2215-0366(21)00243-1. doi: 10.1016/S2215-0366(21)00243-1

26. Fernández-Abascal B, Suárez-Pinilla P, Cobo-Corrales C, Crespo-Facorro B, Suárez-Pinilla M. In- and outpatient lifestyle interventions on diet and exercise and their effect on physical and psychological health: a systematic review and meta-analysis of randomised controlled trials in patients with schizophrenia spectrum disorders and first episode of psychosis. Neurosci Biobehav Rev. (2021) 125:535-68. doi: 10.1016/j.neubiorev.2021.01.005

Conflict of Interest: The authors declare that the research was conducted in the absence of any commercial or financial relationships that could be construed as a potential conflict of interest.

Publisher's Note: All claims expressed in this article are solely those of the authors and do not necessarily represent those of their affiliated organizations, or those of the publisher, the editors and the reviewers. Any product that may be evaluated in this article, or claim that may be made by its manufacturer, is not guaranteed or endorsed by the publisher.

Copyright (C) 2021 Ventriglio, Petito, Castaldelli-Maia, Torales, Sannicandro, Milano, Iuso and Bellomo. This is an open-access article distributed under the terms of the Creative Commons Attribution License (CC BY). The use, distribution or reproduction in other forums is permitted, provided the original author(s) and the copyright owner(s) are credited and that the original publication in this journal is cited, in accordance with accepted academic practice. No use, distribution or reproduction is permitted which does not comply with these terms. 